\title{
1. Historischer Hintergrund
}

Der Fall Hutten markiert einen der Eckpunkte des Forschungsdiskurses über die frühe Geschichte der Provinz Venezuela, die in ihren ersten Jahrzehnten (1528-1556) unter der Statthalterschaft der oberdeutschen Handelsgesellschaft der Welser ${ }^{1}$ aus Augsburg stand. Dieser Finanzdynastie, die über ein weit verzweigtes Netz von Faktoreien in ganz Europa und Übersee verfügte, hatte Kaiser Karl V. im Jahr 1528 die Erschließung und Eroberung der Provinz in einem Vertrag ${ }^{2}$ zugesprochen. Der fränkische Reichsritter Philipp von Hutten hatte sich 1534 in den Dienst der Welser gestellt und kam 1535 nach Coro, einer stadt ${ }^{3}$ an der Nordküste des Kontinents, um zunächst im Grad eines Hauptmanns an der entrada (einem »Entdeckungs«- und Eroberungszug) des damaligen Gouverneurs Georg Hohermuth von Speyer teilzunehmen. In seiner Funktion als Generalkapitän - militärischer Oberbefehlshaber - war er einer der Amtsträger, die diese sogenannte >deutsche Epoche der Welser der Provinz Venezuela prägten.

Die Erwartung war groß, auf ihrer ersten entrada ins Landesinnere von Venezuela ein Goldreich erobern zu können, wie im Falle Francisco Pizarros, dessen Unterwerfung des Inkareiches im Jahr 1534 zur feierlichen Übergabe des kaiserlichen Fünften an den spanischen Monarchen in Toledo führte, der Hutten vermutlich persönlich beigewohnt hatte. ${ }^{4}$ Doch der erhoffte Goldfund blieb aus, und Philipp von Hutten und Georg Hohermuth von Speyer kehrten im Jahr 1538 zwar mit leeren Händen, aber neuen Hoffnungen auf einen Goldfund nach Coro zurück. Sofort begann Hutten mit der Planung einer eigenen entrada, zu der er nach seiner Ernennung zum Generalkapitän mit etwa 100 Mann in Begleitung seines Hauptmanns Bartholomäus Welser des Jüngeren ${ }^{5}$ am 1. August 1541 aufbrach. Hutten und Welser sollten allerdings nicht lebend zurückkehren; sie wurden im Mai 1546 auf dem Rückzug nach Coro von ihrem spanischen Rivalen, dem Gouverneur ${ }^{6}$ Juan de Carvajal, enthauptet. Wie die Zeugenaussagen im späteren Kriminalprozess gegen Carvajal übereinstim- 
mend darlegen, wollte Hutten seinen Herren, den Welsern und dem König, über die Ergebnisse seiner entrada Bericht erstatten. Dazu hätte er nach Coro zurückkehren müssen, was ihm Carvajal wiederum verweigerte. Daraufhin kam es zu verbalen und kämpferischen Auseinandersetzungen; es wurde ein Friedensvertrag ausgehandelt und von den Beteiligten beider Parteien unterzeichnet. Trotzdem überfiel Carvajal Huttens Lager und nahm diesen und Welser sowie zwei weitere Männer der Truppe gefangen. Den Schlusspunkt setzte Carvajal, indem er die Vier Mitte Mai 1546 enthaupten ließ.

Als Juan Pérez de Tolosa nur wenige Wochen später, am 10. Juni 1546, in Coro ankam, um den Einsatz zur Durchführung des regulär angesetzten juicio de residencia, ein Verfahren zur Überprüfung der Erfüllung der Capitulación durch die Welser, anzutreten, erfuhr er von den blutigen Kämpfen und Auseinandersetzungen in der Provinz. Er befragte mehrere Zeugen, die aus Carvajals Lager im Landesinneren nach Coro zurückgekommen waren. Da er die Gerüchte bestätigt fand, leitete er einen Kriminalprozess ein. In dessen Verlauf stellte er eine Truppe zusammen, mit der er nach El Tocuyo in Juan de Carvajals Lager zog, ihn verhaftete und schließlich zum Tode verurteilte. Er sollte am Schweif eines Pferdes zum Galgen geschleift und dort gehängt werden. Die Prozessakten bezeugen am 27. Oktober 1546, dass das Urteil nach Carvajals Geständnis vom 17. September 1546 am Fuß des Galgens, bei dem der Angeklagte die alleinige Schuld auf sich nahm und andere, ursprünglich ebenfalls angeklagte Mitstreiter vollständig entlastete, vollstreckt worden war.

Diese knappe Darstellung der Ereignisse soll zur Orientierung der Lesenden dienen. Das Wissen, das ihr zugrunde liegt, stammt aus den Texten unseres Korpus und aus der historiographischen Forschung, die unter anderem jene Texte als Quellen verarbeitet haben. Im Folgenden soll der Stand der Forschung dargestellt werden, die sich mit Philipp von Hutten und seiner »Ermordung ${ }^{7}$ befasst hat (vgl. Friede 1961; P. v. Hutten 1999; Schmitt/Simmer 1999; Simmer 2000; Denzer 2005). Die Forschungen zu den einzelnen Texten des Korpus, an welche die Analysen anschließen, werden in den entsprechenden Kapiteln dargestellt.

Juan Friedes umfassende Aufarbeitung der Welser-Epoche (vgl. Friede 1961: Los Welser en la conquista de Venezuela) gilt nach Denzer »in Deutschland als das lateinamerikanische Standardwerk zum Venezuelaunternehmen der Welser« (2005: 267). 1901 in Russland geboren, sei Friede, »offenbar ein Nachkomme deutscher Einwanderer« (ebd.), im Jahr 1927 nach Kolumbien eingewandert, 
wo er bis zu seinem Tod 1990 gelebt habe. In seinem Werk möchte er nach eigenen Angaben die Rolle der »alemanes « (der >Deutschen«) in der Eroberung und Kolonisation von Venezuela und Kolumbien »klären«, »nicht verteidigen« (ebd.: 7). Die Handlungsweise der deutschen Regenten würde sich nicht so stark von den Übrigen - Spanischen - abheben, wie dies die kolonialen Chronisten, aber auch einige moderne Historiker aus politischen, ideologischen oder nationalistischen Gründen behaupteten (vgl. ebd.: 8). Diese einleitenden Hinweise machen klar, dass sich im Venezuela der 1960er Jahre eine Diskussion um die Tatsache entzündet hatte, dass >Ausländer < - will heißen: Nichtspanier - an der conquista beteiligt waren. Dieses in den damaligen $\mathrm{Na-}$ tionalismus der venezolanischen und kolumbianischen Diktaturen der 1940er und 1950er Jahre einzuordnende Moment floss in Friedes Untersuchung ein. Er erzählt das »Drama en la selva: Hutten y Carvajal ${ }^{8}$ (Friede 1961: 375) im wörtlichen Sinn als >Drama< nach - als ein Theaterstück mit vielen direkt wiedergegebenen Figurenreden, die er den Akten des Prozesses gegen Carvajal teilweise unhinterfragt und eins zu eins übernimmt. Wie aber die vorliegende Untersuchung (in diesem Fall das Kapitel 3) zeigen wird, handelt es sich bei den Prozessakten um höchst komplexe sprachliche Gebilde, die keinesfalls die >Wahrheit< abbilden (können). Friede beurteilt abschließend das Verhalten Carvajals ohne nähere Begründung: »No hay duda de que, desde el principio, todo lo tramado por Carvajal fue hecho con intención de deshacerse más fácilmente de un hombre, que, llegado a Coro, podría serle fatal. «9 (Ebd.: 398) Eine Rekonstruktion der Ereignisse einer Epoche aufgrund von Dokumenten, die von ihrer Anlage her nicht die >Wirklichkeit abbilden können, muss auf mehr als wackligen Beinen stehen. Sie scheint jedoch einer Art Texttradition $\mathrm{zu}$ entspringen, welche die Geschichtsschreibung der mittleren Jahrzehnte des 20. Jahrhunderts prägte.

Ähnliche Züge wie das Werk Juan Friedes zeigen auch die Arbeiten von Bruder Nectario María (1968 [1952]). Dieser hatte Ende der vierziger Jahre des 20. Jahrhunderts die Gründungsgeschichte der Stadt Barquisimeto in den Archiven erforscht. Dabei ist ist eine Art Biographie ihres Gründers Juan de Villegas herausgekommen, eines Protagonisten, der auch im Fall Hutten eine gewisse Rolle spielt: Historia de la fundación de la ciudad de Nueva Segovia de Barquisimeto a la luz de los documentos de España y de Venezuela, ${ }^{10}$ so der Titel des Buches. Wie Friede stellt auch Nectario María Behauptungen auf, ohne sie zu begründen, geschweige denn offensichtliche Widersprüche zu klären. Beispielsweise schreibt er, dass Carvajal allein auf die »teuflische Idee« (ebd: 52) gekommen sei, Hutten bei einem Überraschungsangriff zu töten, nachdem 
dieser nach der Unterzeichnung des Friedensvertrags Richtung Coro losgezogen sei. In einer Fußnote gibt Nectario María ohne weiteren Kommentar an, dass Carvajal vor Gericht ausgesagt habe, Pedro de Limpias und Sebastián de Almarcha hätten Carvajal auf die Idee gebracht, Hutten zu überfallen (vgl. Nectario María 1968: 52). Diesen Widerspruch zu seiner eigenen Sichtweise müsste Nectario María zumindest benennen und auch Überlegungen anführen, die ihn zu seinem, der Darstellung Carvajals widersprechenden Schluss geführt haben. Auch fügt er in ästhetisch-literarisch anmutender Weise Beschreibungen ein, die so nicht in den Quellen vorkommen: »En la sierra, camino de Coro, a tiempo en que todos descansaban en sus hamacas, a la sombra de árboles frondosos, [Carvajal] cayó sobre ellos « ${ }^{11}$ (ebd.: 53). Als Nectario María die Szene am Fuß des Galgens nacherzählt, spricht er von Carvajals »voz humilde y trémula « ${ }^{12}$ (ebd.: 57), die dieser gemäß Zeugnis des Schreibers Juan de Quincoces de Llana erhoben haben soll. Diese Beschreibung der Stimme Carvajals ist eine Erfindung Nectario Marías, die dieser aber nicht als solche kenntlich macht. Insgesamt zeichnet sich die lateinamerikanische Geschichtsschreibung des 20. Jahrhunderts also durch eine starke Prägung durch zeitgenössische politische Strömungen aus. Nach Denzer (vgl. 2005: 272f.) wird die Epoche der Welser in Venezuela bis heute ausgeblendet. ${ }^{13}$

Die deutsche Geschichtsschreibung arbeitet dagegen eher editionsgeschichtlich. Eberhard Schmitt ediert im Jahr 1999 in Zusammenarbeit mit Friedrich Karl von Hutten, einem Nachfahren von Philipp, erstmals die Briefe, die Letzterer aus Venezuela geschrieben hatte, als Gesamtheit (vgl. Hutten, P. v. 1999: Das Gold der Neuen Welt. Die Papiere des Welser-Konquistadors und Generalkapitäns von Venezuela Philipp von Hutten 1534-1541). Einige der Briefe wurden erst kurz zuvor wiederentdeckt. ${ }^{14}$ Zusammen mit Götz Simmer gab Schmitt im selben Jahr eine Sammlung der aus ihrer Sicht wichtigsten Dokumente in deutscher Übersetzung heraus, die den Tod des fränkischen Reichsritters nachzeichnen (vgl. Schmitt/Simmer 1999: Tod am Tocuyo. Die Suche nach den Hintergründen der Ermordung Philipps von Hutten 1541-1550). Beide Ausgaben von 1999 sind in erster Linie Editionen, versehen mit zahlreichen Erläuterungen und Erklärungen zentraler Begriffe, ergänzt durch ein sehr nützliches Personenverzeichnis, in dem alle verfügbaren Informationen $\mathrm{zu}$ einzelnen Figuren zusammengetragen werden.

Götz Simmer (vgl. 2000) widmet neun Kapitel des Teils VI seines Buches Gold und Sklaven: Die Provinz Venezuela während der Welser-Verwaltung (1528-1556) den Ereignissen um die Auseinandersetzung zwischen den beiden Konquistadoren (vgl. ebd.: 534-605), indem er sämtliche Quellen minutiös inhaltlich 
aufarbeitet und in ähnlicher Weise wie Juan Friede zu einer einheitlichen Erzählung zusammengefügt. Informationslücken und inhaltliche Widersprüche werden mit Aussagewerten anderer Quellen zu klären versucht. Insgesamt kommt er zu dem Schluss, dass das Urteil gegen Carvajal gerechtfertigt war und dass im Kriminalprozess gegen ihn »juristisch hinreichend klar bewiesen worden « (ebd.: 593) sei, dass die von ihm begangenen Straftaten die verhängte Strafe rechtfertigen würden. »Die gesamte Untersuchung [des Kriminalprozesses gegen Carvajal; SG] allerdings war deutlich auf die Herstellung des Rechtsfriedens hin orientiert, nicht auf Gerechtigkeit.« (Ebd.) Richter Juan Pérez de Tolosa habe in der »von Konflikten zerrissenen Provinz integrativ wirken « wollen und deshalb von "verschärften Untersuchungen und Verfolgungen im Kreis der Offiziere von El Tocuyo« abgesehen (ebd.: 592f.). Den damit erzeugten Widerspruch, dass das Urteil gerechtfertigt war und gleichzeitig andere Beschuldigte unbehelligt geblieben waren, löst Simmer nicht auf.

Jörg Denzer (vgl. 2005) seinerseits geht in drei Unterkapiteln seines Buches Die Konquista der Augsburger Welser-Gesellschaft in Südamerika 1528-1556 Huttens entrada nach. Er kommt dabei zum Schluss, dass die letzte entrada unter den Welsern zur »Abwicklung der Konkursmasse« (ebd: 163) gedient habe. Philipp von Hutten habe als Vertreter des Handelshauses der Welser, »das keine Lobby mehr in Venezuela besaß (ebd.: 187), auf keine Loyalität mehr zählen können. Denzer interpretiert die "Ermordung Huttens und seiner Begleiter « als bezeichnend für das Zu-Ende-Gehen der Welser-Ära in Venezuela und ordnet das "Verschwindenlassen einer ohnehin bereits verloren geglaubten Expeditionstruppe (ebd.) als Bestreben Carvajals ein, seine Macht zu erhalten. Der Mord habe auch »nur in dem von den Welsern hinterlassenen Machtvakuum geschehen " (ebd.: 193) können: Nach Erkenntnissen Denzers zog sich die Handelsgesellschaft ab 1540 aus Venezuela zurück, als die Krone die Zusammenarbeit mit privaten Konquistaunternehmern abgebrochen habe (vgl. ebd.: 192). In jenem Jahr sei mit Cundinamarca, dem »Nuevo Reino de Granada «(sNeues Königreich von Granada‘), die letzte lohnende Erwerbung in spanischen Überseebesitz eingegangen; es handle sich dabei um das "Schlüsselereignis zum Verständnis des Übergangs von der Ära der Konquista zur Ära der Kolonisation « (ebd.: 192). Dieser Politikwechsel der Krone habe zur »Entmachtung der Konquistadoren" (ebd.: 152) geführt, die unter anderem ihren Ausdruck in den Leyes Nuevas von 1542 gefunden hätten: 
In der als >Leyes Nuevas< (>Neue Gesetze`) bekannten >Real Provisión< (>Königlichen Verfügung`) von 1542 wurden die Rechte der Encomenderos über die von ihnen unterworfenen Indianer stark eingeschränkt, die Encomienda durch das >Repartimiento<, die staatlich gesteuerte und stark eingeschränkte Zuteilung indianischer Arbeitskräfte, ersetzt. Allgemein wurde der weiteren Erschließung und Kultivierung des Kontinents durch staatliche und kirchliche Stellen gegenüber der von Privatunternehmern der Vorrang gegeben. Mit den Leyes Nuevas wurde den Konquistadoren eine von diesen als standesgemäß empfundene Lebenshaltung, die auf der Ausbeutung der Indianer beruhte, unmöglich gemacht und ihr politischer Einfluss gedämmt. (Ebd.: 154)

Das Jahr 1540 kann also gemäß dieser Darstellung als "plötzliche Beendigung der Konquista durch die Krone« (ebd.: 155) gesehen werden, in deren Folge es zu zahlreichen kleineren und größeren Unruhen, Rebellionen und Aufständen gekommen ist, über die aber wegen der spanischen Zensur nur wenig bekannt wurde. Der >Mord an Philipp von Hutten und seinen Mitstreitern fiel also in eine Zeit, in der es in der Konquistadorenschicht zu großen Umwälzungen gekommen ist, welche die Handlungsweise der beiden Gegner Hutten und Carvajal mit Sicherheit mit beeinflusst haben.

Die Produktion der Texte, die der vorliegenden Studie zugrunde liegen, fällt gemäß dieser Darstellung also in den Übergang von der Phase der >Entdeckung in die der Kolonisation. Entsprechend dieser These und der Annahme, dass es sich um einen der »historisch wichtigen Übergänge« (im Sinne Pratts: »important historical transitions«, Pratt 2008: 4) in der Reiseliteratur handelt, wird das untersuchte Korpus in diesem Buch als Text der Kolonisation gelesen. Pratt schreibt, dass solche wichtigen historischen Übergänge

alter the way people write, because they alter people's experiences and the way people imagine, feel and think about the world they live in. These shifts in writing, then, will tell you something about the nature of the changes. Such shifts in writing, if they are historically profound, affect more than one genre. (Ebd.)

Mit anderen Worten: Sind die Übergänge textwirksam, haben sie eine Periodisierung der kolonialen Texte zur Folge. Eine ähnliche Annahme trifft auch Rabasa (vgl. 1993: 15f.), wenn er feststellt, dass die verschiedenen Momente und Situationen der kolonialen Epoche jeweils eigene genrehafte Texte ermöglicht und hervorgebracht hätten. Während die Kolumbus-Briefe als Text 
der »Entdeckung« die Erfindung (vgl. der Titel seines Buches: Inventing America: Spanish Historiography and the Formation of Eurocentrism) der Neuen Welt eröffneten, legte Cortés den Grundstein der Texte der Entdeckung und Eroberung.

Texte der Kolonisation - also der Phase der Konsolidierung und des Übergangs zur Normalität der kolonialen Herrschaft - müssten dementsprechend auch als solche markiert und erkennbar sein. Solche Markierungen werden in den Kapiteln 4 bis 6 herausgearbeitet. Zunächst soll die Studie jedoch in die Debatten der Kolonialforschung eingeordnet werden. 
